\title{
Comorbidity among Hospitalized Patients with Chronic Obstructive Pulmonary Disease in a Teaching Hospital, West Java Indonesia
}

\author{
Dini Qurrotu Aini, ${ }^{1}$ Hendarsyah Suryadinata, ${ }^{2}$ R.B. Soeherman Herdiningrat ${ }^{3}$ \\ ${ }^{1}$ Faculty of Medicine Universitas Padjadjaran, ${ }^{2}$ Department of Internal Medicine, Faculty of \\ Medicine Universitas Padjadjaran/Dr. Hasan Sadikin General Hospital Bandung, ${ }^{3}$ Department of \\ Anatomy and Cell Biology Faculty of Medicine Universitas Padjadjaran
}

\begin{abstract}
Background: One of the most important causes that can increase the risk of hospitalization and death in chronic obstructive pulmonary disease is comorbidity. The aim of this study was to identify the proportion of comorbidity among hospitalized patients with chronic obstructive pulmonary disease in a teaching hospital, West Java, Indonesia, from January to December 2012.

Methods: A descriptive study was conducted from September to November 2013 in Internal Medicine Department of Dr. Hasan Sadikin General Hospital Bandung. The study used 107 medical records that consisted of data about patients with chronic obstructive pulmonary disease (COPD) who were hospitalized between January to December 2012. The medical records were collected using simple random sampling. Variables identified in this study were characteristics of the patients (age, sex, and smoking history) and comorbidity events. Comorbidity events were coronary artery disease (CAD), lung cancer, diabetes mellitus (DM) type 2, anemia, dyslipidemia, osteoporosis, depression, pulmonary artery hypertension (PAH), and hypertension. Age variable was divided into 3 categories with interval 20 years. All collected data were presented in frequency distribution.

Results: Most of the patients in this study were 50-69 years old, male, and had smoking history. The highest proportion of comorbid condition was hypertension, followed by anemia and coronary artery disease.

Conclusions: Patients with COPD have one or more other diseases (comorbidity). Three most frequent comorbidities are hypertension, anemia, and coronary artery disease. [AMJ.2015;2(4):485-91]
\end{abstract}

Keywords: Chronic obstructive pulmonary disease, comorbidity, hospitalized patient

\section{Introduction}

Chronic obstructive pulmonary disease (COPD) is a lung disease characterized by chronic airflow limitation that interferes with normal breathing and is not fully reversible. World Health Organization (WHO) stated that in 2004, 64 million people had COPD and 3 million of them passed away caused by COPD. WHO also predicted that in 2030, COPD will become the third leading cause of death. ${ }^{1}$ Among 12 Asia-Pacific region countries, Indonesia became the fourth leading prevalence of COPD with prevalence about $5.6 \%{ }^{2}$ The COPD is a preventable and treatable disease and related to a systemic inflammatory response that may contribute to individual severity. ${ }^{3}$

In a majority of cases, increase in systemic inflammatory components may underlie many of the multiple comorbidity seen in people with COPD. ${ }^{4}$ Fabbri ${ }^{5}$ categorizes these comorbidity, caused by systemic inflammation, as Chronic Systemic Inflammatory Syndrome (CSIS). The most recognized comorbidities are hypertension, coronary artery disease (CAD), lung cancer, osteoporosis, anemia, diabetes mellitus (DM) type 2, depression, and pulmonary artery hypertension (PAH). Comorbidity in patients with COPD must be identified and evaluated because it can increase the risk of hospitalization and mortality. ${ }^{6,7}$ The objective of this study was to identify the proportion of comorbidity among hospitalized patients with COPD in a teaching hospital, West Java, Indonesia

\section{Methods}

A descriptive study was conducted from September to November 2013 at Internal

Correspondence: Dini Qurrotu Aini, Faculty of Medicine, Universitas Padjadjaran, Jalan Raya Bandung-Sumedang Km.21, Jatinangor, Sumedang, Indonesia, Phone: +6285691236913 Email: diniqurrotuaini@gmail.com 
Medicine Department in Dr. Hasan Sadikin General Hospital Bandung. The study used medical records that consisted of data about patients with chronic obstructive pulmonary disease (COPD) who were hospitalized from January to December 2012. Patients who had COPD diagnosis either as the primary or secondary diagnosis on medical records were included, and patients whose medical record were not found and had no information about age, sex, and smoking history were excluded. Research medical records for this study were collected using simple random sampling.

During this study, from 337 medical records, only, 183 were selected, but only 153 patients whose medical record were found and identified having a COPD diagnosis as primary or secondary diagnosis on medical records. Moreover, 46 medical records were excluded because they had incomplete data on age, sex, or smoking history. Therefore, the total medical records which could be analyzed in this study were 107 medical records.

Variables identified in this study were characteristics of the patients and comorbidity events. Characteristic variables consisted of age, sex, and smoking history, whereas comorbidity events were coronary artery disease (CAD), lung cancer, diabetes mellitus (DM) type 2, anemia, dyslipidemia, osteoporosis, depression, pulmonary artery hypertension (PAH), and hypertension. Age variable was divided into 3 categories with interval 20 years. CAD, lung cancer, depression, osteoporosis, and PAH were determined based
Table 1 Characteristics of the COPD Patients

\begin{tabular}{lc}
\hline \multicolumn{1}{c}{ Characteristics } & $\mathbf{n}(\%)$ \\
\hline Age (yrs.) & \\
$<50$ & $8(7.48 \%)$ \\
$50-69$ & $54(50.47 \%)$ \\
$\geq 70$ & $45(42.06 \%$ \\
Sex & \\
Male & $93(86.92 \%)$ \\
Female & $14(13.08 \%)$ \\
Smoking History & \\
Yes & $95(88.79 \%)$ \\
No & $12(11.23 \%)$ \\
\hline
\end{tabular}

on diagnosis on medical records. DM type 2 was confirmed according to ADA 2010 criteria. Anemia was confirmed with Indonesian Health Department criteria. Dyslipidemia was confirmed based on National Cholesterol Education Program (NCEP) Adult Treatment Panel (ATP) III criteria and hypertension was confirmed based on Joint National Committee (JNC) 7 criteria.

All collected data were presented in frequency tabulation and percentage. The study was approved by Health Research Ethics Committee, Faculty of Medicine Universitas Padjadjaran (No. 210/UN6. C2.1.2.KEPK/2013). Collected data from medical records were kept confidentially and

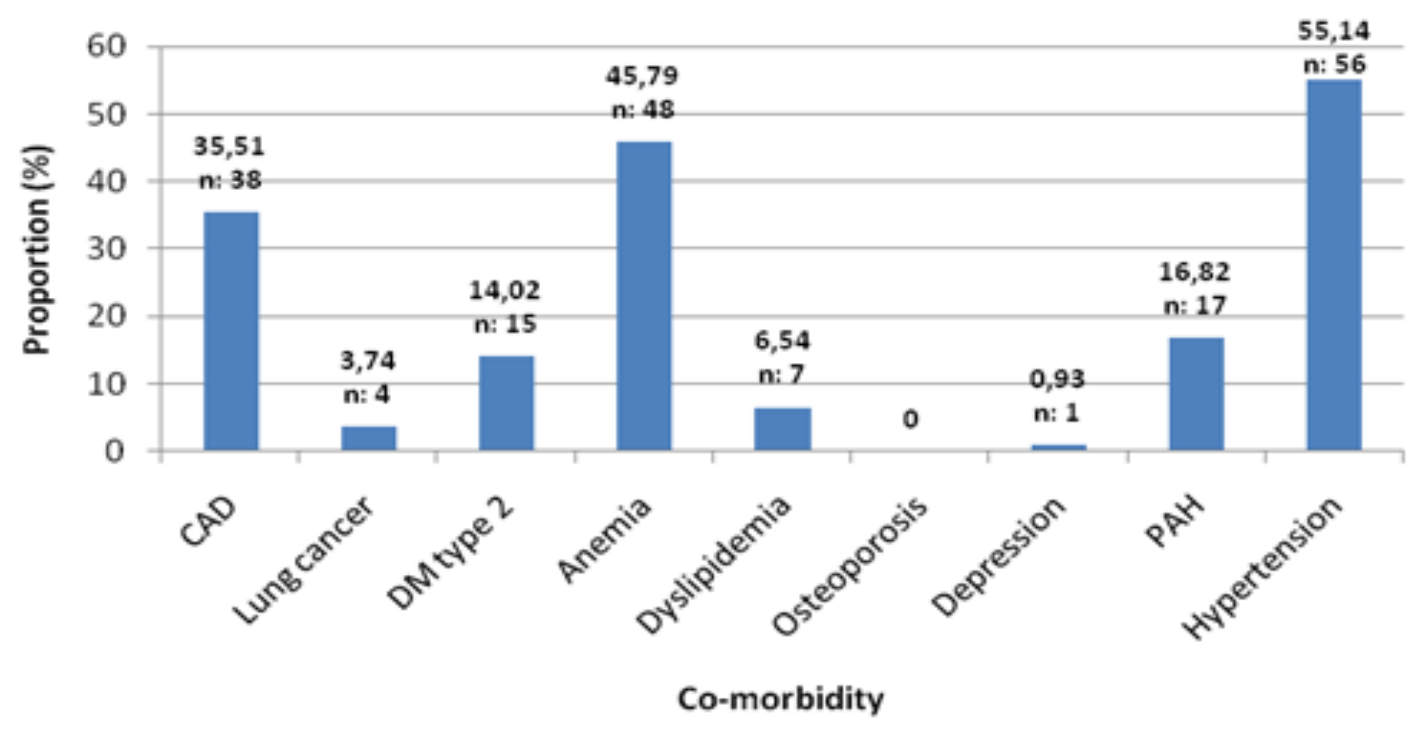

Figure 1 Proportion of Comorbidity 


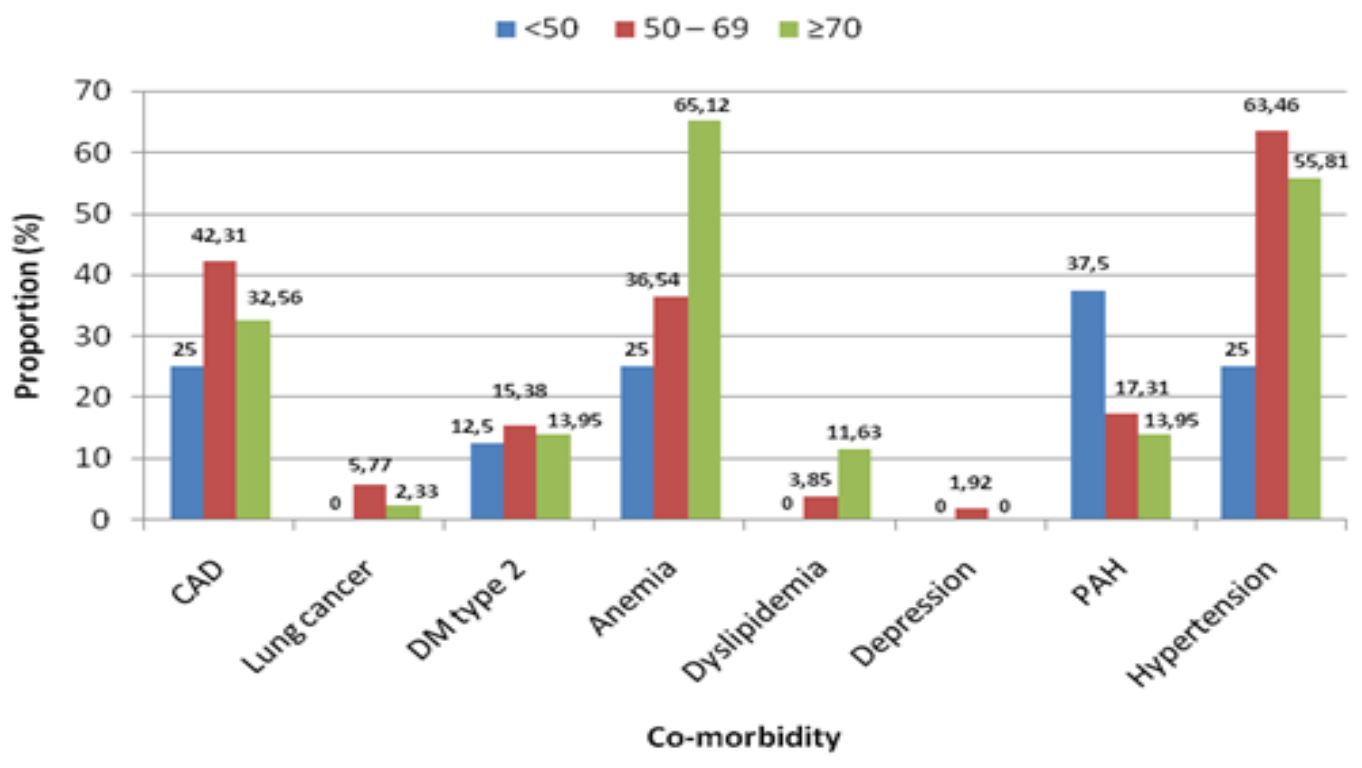

Figure 2 Distribution of Comorbidity according to Age

vanished after the study was conducted.

\section{Results}

Most of patients in this study were 50-69 years old, male, and had smoking history. In this study, various conditions of comorbidity were identified and there was more than one comorbidity in each patient. The highest proportion of comorbid condition was hypertension, followed by anemia and coronary artery disease $(55.14 \%, 45.79 \%$, and $35.51 \%$, respectively).

Pulmonary artery hypertension (PAH) had the highest proportion of comorbity in patients aged under 50 years old, meanwhile hypertension was the highest proportion of comorbity in 50-69 years old patients. Above 70 year old patients, anemia was the highest proportion of comorbidity.

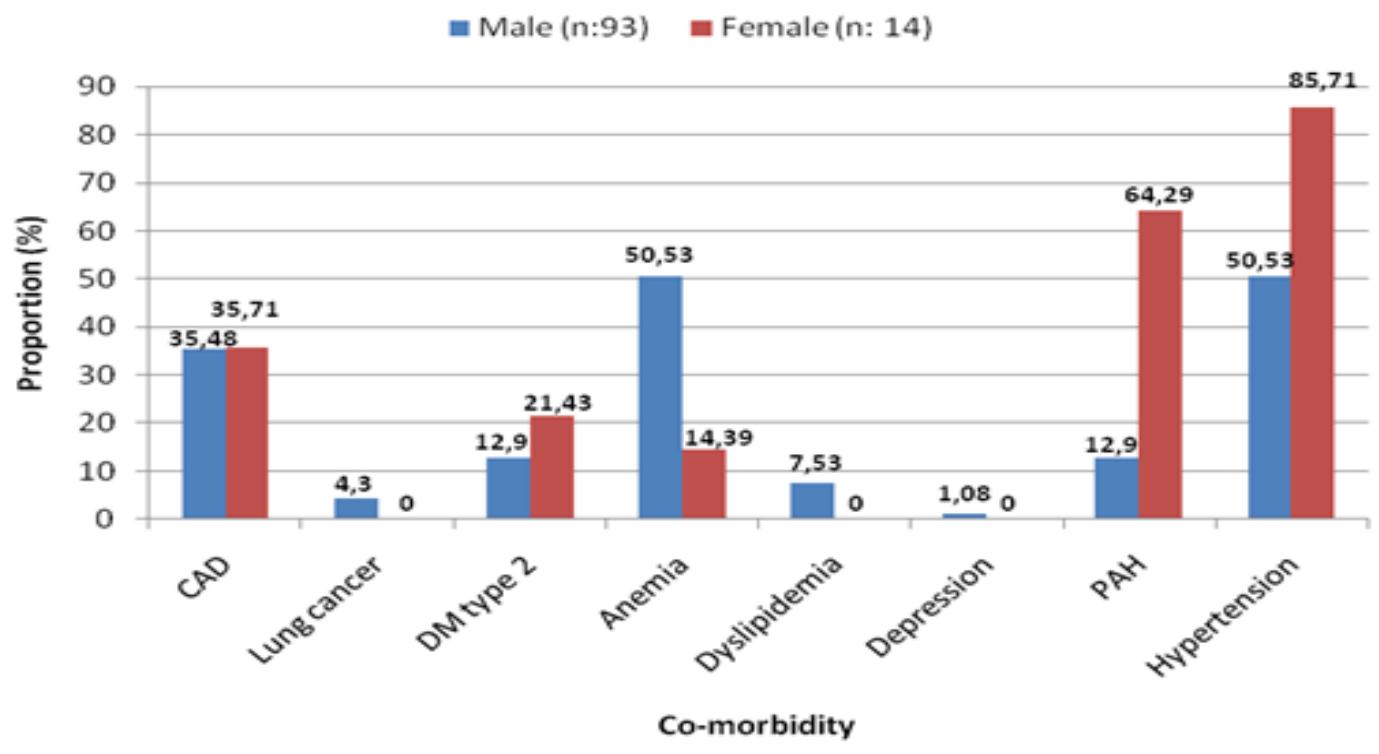

Figure 3 Proportion of Comorbidity according to Sex 


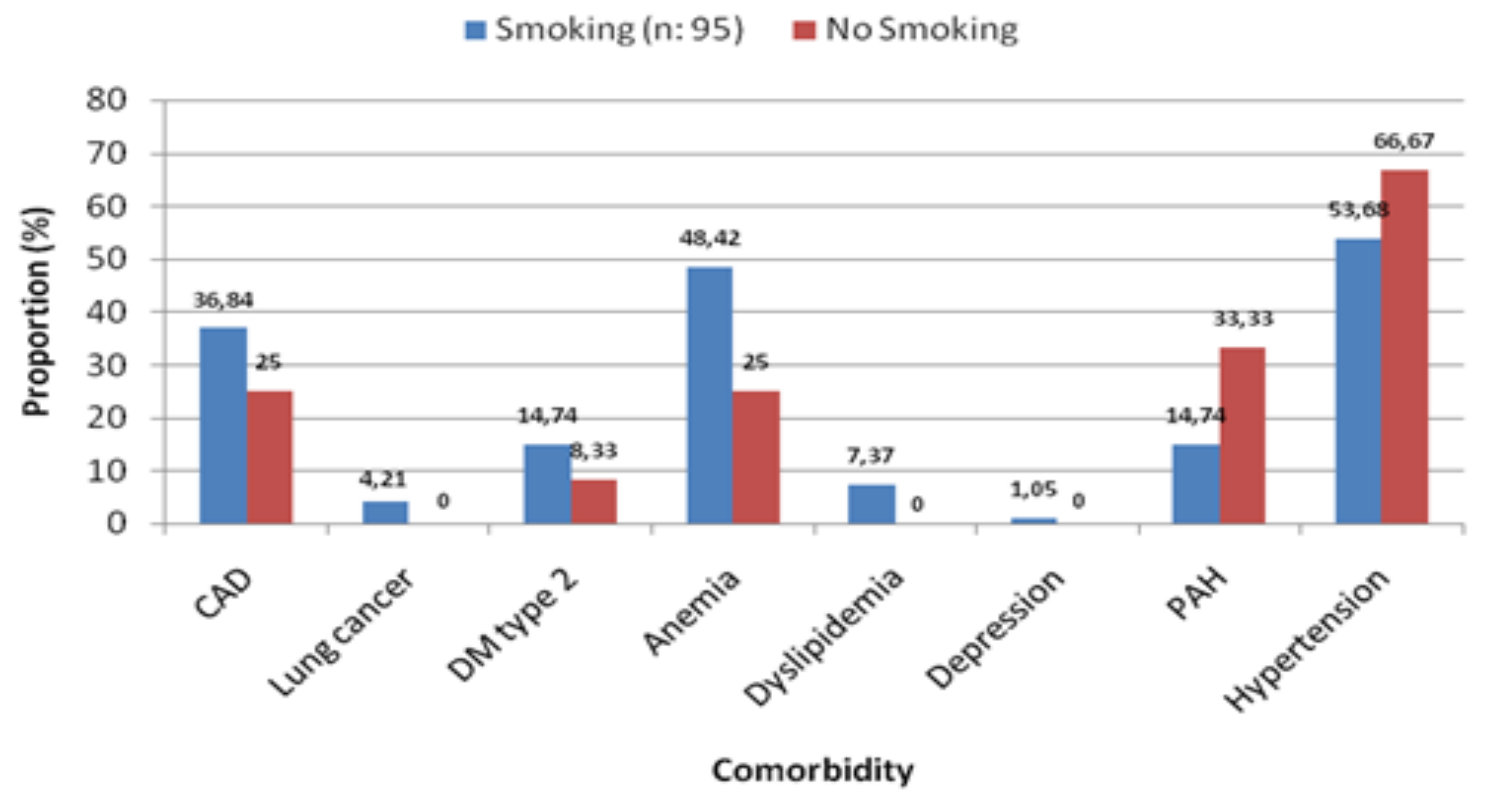

Figure 4 Proportion of Comorbidity according to Smoking History

If divided by sex, proportion of lung cancer, anemia, dyslipidemia, and depression, the number of male patients were higher compared to female patients. In contrary, a higher proportion of DM type 2, PAH, and hypertension in female patients were identified compared to male patients. The proportion of CAD in male subjects was almost same as in female subjects, which were $35.48 \%$ and $35.71 \%$ (Figure 3).

Proportion of CAD, lung cancer, DM type 2 , anemia, dyslipidemia, and depression in COPD patients with smoking history were higher compared to patients without smoking history. Meanwhile, in PAH and hypertension, the proportion was higher in patients without smoking history (Figure 4).

\section{Discussion}

Hospital discharge with primary or secondary COPD was mostly diagnosed with one or more comorbid conditions, including cardiac and pulmonary vascular disease, pneumonia, malignancies, chronic kidney disease, congestive heart failure, gastropathy, and others. Some of the most common comorbid conditions described in association with COPD include coronary artery disease (CAD), lung cancer, diabetes mellitus (DM) type 2, anemia, dyslipidemia, osteoporosis, depression, pulmonary artery hypertension (PAH), and hypertension.

Comorbid condition which had a highest proportion found in this study was hypertension. The proportion was about $55.14 \%$. This result strengthened the prospective cohort study conducted by Huiart8 in which among 5648 patients with COPD, the prevalence is about 50\%.8 Moreover, Mills et al.9 through the study, reported that twothirds of COPD populations have a high arterial stiffness and blood pressure, and in one study conducted by Maninno10 the prevalence of hypertension in COPD patients is $40.1 \%$. The major determinant of increased blood pressure in patients with COPD was large artery stiffness. Arterial stiffness was influenced by both structural and functional aspect of the artery. In structural aspect, impaired lung function and systemic inflammation could cause a premature ageing of vasculature and reduced elastic fiber contents in dermis leading to an arterial stiffness. Whereas, in functional aspect, endothelial dysfunction happened in smoker could influence endothelial dependent vasomotor tone. If stratified by age, the proportion of hypertension in this study was higher in older group. It supports another study which reported that arterial stiffness in patients with COPD increases with age. If stratified by sex, the proportion of 
hypertension was higher in females, contrary with previous study which said that male have a higher risk than female. If stratified by smoking history, the proportion was higher in non-smoking group. It is different from previous study which said that cigarette smoke can make a structural and functional abnormality of vasculature. ${ }^{9}$

Two comorbid conditions with high proportion right after hypertension were anemia and coronary artery disease (CAD). The mechanism why anemia could be occurred in people with COPD was similar to the mechanism in other chronic diseases. The increased level of IL- 6 and TNF- $\alpha$ in COPD patients, led to shortened red blood cell (RBC) survival. Moreover, there was a demand to increase RBC production, but the bone marrow did not respond adequately because of erythropoietin resistance. ${ }^{11}$ The proportion of anemia in this study was $45.79 \%$. Three studies conducted reported that the anemia prevalences in COPD are 23\%, 21\%, and $15 \%{ }^{6,11}$ Anemia could be caused by many conditions or diseases, including bleeding, malignancies, chronic kidney disease (CKD). Those conditions or diseases were found in subjects in this study and there was no control over those conditions. This might be a reason why the proportion of anemia in this study was higher than other studies. If stratified by sex and age, the proportion of anemia was higher in male and increased with age. This result supports a study conducted by Yohannes ${ }^{12}$ which said that anemia in COPD patients is more likely in male and older. If stratified by smoking history, this result also supports that study which said that cigarette smoking has a carboxyhemoglobin effect causing anemia. ${ }^{12}$

Holguin $^{13}$ explained that CAD prevalence in COPD is $15 \%$ and the proportion of CAD found in this study was $37.25 \%$. In people with COPD, raising in inflammatory mediators such as TNF- $\alpha$ and IL-6, could increase the level of C-Reactive Protein (CRP). CRP increased LDL uptake from circulation by macrophage. Moreover, CRP could adhere directly on arterial wall and interacted with inflammatory mediator forming foam cells which is an atherogenic plaque. Beside CRP, raising in fibrinogen level could increase blood viscosity and then contributed in atherosclerosis process.7 If stratified by smoking history and age, CAD was more likely found in smoking group and in 50-69 year old group. Sex, male, and female subjects had the same proportion about 35\%. Besides, COPD patients had several other risk factors that might contribute to development of CAD such as smoking. Smoking habit is clearly a risk factor for both CAD and COPD, and can potentially interfere with our result because most of the subjects in this study had a smoking history. ${ }^{10}$

Osteoporosis became the lowest-proportion co-morbid with proportion about $0.00 \%$. This result was different compared to other studies. According to Lidwien ${ }^{14}$ the prevalence of osteoporosis based on dual-energy x-ray absorptiometry DXA and Spinal X-ray examination are $23.6 \%$ and $36.5 \%$. However, COPD patients have several other risk factors that might contribute to development of osteoporosis such as older, poor mobilization, smoking, poor nutrition, low BMI, and using oral or inhalation corticosteroid. Furthermore, COPD is a direct risk factor for osteoporosis through systemic inflammation process. ${ }^{15}$ In this study, the result was $0.00 \%$. This is probably due to a fact that in general, osteoporosis is an asymptomatic disease and BMD examination is rarely done in clinical practice if there is no symptom or indication. Therefore, a further study with primary data is needed.

The proportion of DM type 2 reported from this study was $14.71 \%$. It supports the previous study in which the prevalence of DM type 2 in COPD patients is between 1.6$16 \% .{ }^{6}$ Based on several studies, diabetes in COPD is caused by pro-inflammatory cytokine (TNF- $\alpha$ and IL-6) which can block signal through insulin receptor; as the result, insulin resistance is occurred. Therefore, COPD patient has a higher risk to have DM type 2. If stratified by age, the result supports a study conducted by Mannino ${ }^{10}$ The study found that increasing age, a higher BMI, lower education status, and male sex relate to a higher risk of diabetes. Contrarily, this study result is not in accordance with that study if compared by sex and smoking history because the author explained that there was no association between them.

Lung cancer and COPD share a same risk factor, which is tobacco smoking. This statement is supported by this study because most of both COPD patients and lung cancer patients were smoking. In this study, the proportion for lung cancer was $3.74 \%$. De Torres et al16 in his cohort study reported that $215(8.58 \%)$ cases of lung cancer in 2507 patients with COPD and conclude that older age is the independent risk factor for lung cancer. That study is supported by this study because in this study, lung cancer developed in patient in 50-69 years old group and $\geq 70$ year old 
group. In one study, incidence of lung cancer in mild and moderate airflow obstruction is 6.8\% for non-smoker and $10.8 \%$ for smoker. Barnes and Celli,15 through their study, explained that female has a higher risk to develop COPD and lung cancer as a result from hormonestimulated metabolism of carcinogen within cigarette. Male patients with COPD were patients who had a lung cancer in this study. This contrary finding may be caused by a fact that in our study, the subjects are dominated by males. The total of female subjects was too small to represent the female population.

In this study, pulmonary arterial hypertension was found about 16.82\%. Meanwhile, in most previous studies, the prevalence of pulmonary hypertension is ranging from $30-70 \%{ }^{17,18}$ The proportion of PAH in this study was lower than several previous studies. This was probably due to pulmonary artery pressure (Ppa) data that were not found in medical records and the definition of PAH. This study was mostly made based on presence of cor pulmonale in the subjects. Nine of eighteen subjects with pulmonary hypertension were in 50-69 year old group, supporting a study conducted by Joppa ${ }^{18}$ which said that the mean of age is 68 years old. Among 18 cases of pulmonary hypertension in this study, 13 male participated and 14 patients had smoking history. It also supports the previous study.

In this study, only one case $(0.93 \%)$ of depression was found and it was male who had a smoking history, and in 50-69 year old group. It is different from one review article which concluded that the prevalence of depression is $19-42 \% .{ }^{15}$ This was probably caused by a diagnosis which was determined only from medical records and the subjects which were not screened for depression. Seven cases of dyslipidemia were reported from this study, with proportion about 6.54\%. The proportion of dyslipidemia was higher in male and smoking group, and the proportion increased with age.

There are several limitations in this study. First, COPD in this study was obtained from diagnosis on medical records in which most of them were defined clinically, not based on spirometry as a gold standard for COPD. Second, this study used secondary data from medical records which had limited information and could not be explored. Some of them also had incomplete data causing difficulty in analyzing data and made the sample less represent the population. Then, most of comorbidities were determined only from diagnosis on medical records causing there was a possibility to have a false negative result. Third, other potential condition such as bleeding, malignancies which could interfere the result was not excluded. Therefore, a further study which used primary data as the source of data and spirometry data as inclusion criteria is needed.

Based on the discussion in this paper, it is concluded that the proportion of several comorbidities in COPD patients are high. Three most frequent comorbidities are hypertension, anemia, and coronary artery disease. The distributions based on age, sex, and smoking history are obtained in this study. Comorbidities in patients with COPD can increase the risk of hospitalization and the mortality rate. Therefore, in order to reduce the risk of hospitalization and the rate of mortality, identifying the presence of co-morbidities in COPD patients based on patient's age, sex, and smoking history should be routinely performed.

\section{References}

1. World Health Organization. Chronic obstructive pulmonary disease (COPD). 2013 [cited 2013 February 7]; Available from: http://www.who.int/respiratory/ copd/en/.

2. Tan W, Seale J, Charaoenratanakul S, De Guia T, Ip M, Mahayiddin A, et al. COPD prevalence in 12 Asia-Pacific countries and regions: Projections based on the COPD prevalence estimation model. Respirology. 2003;8(2):192-8.

3. GOLD. Global strategy for the diagnosis, management and prevention of COPD. Global Initiative for Chronic Obstructive Lung Disease (GOLD); 2001 [cited 2013 March 2]. Available from: http://www. goldcopd.org/.

4. Yawn B, Kaplan A. Co-morbidities in people with COPD: a result of multiple diseases, or multiple manifestations of smoking and reactive inflammation. Prim Care Respir J. 2008;17(4):199-205.

5. Fabbri LM, Rabe KF. From COPD to chronic systemic inflammatory syndrome?. Lancet. 2007;370(9589):797-9.

6. Chatila WM, Thomashow BM, Minai OA, Criner GJ, Make BJ. Comorbidities in chronic obstructive pulmonary disease. Proc Am Thorac Soc. 2008;5(4):549-55.

7. Masna IAK, Kusmana D, Antariksa B. Effect of systemic inflammation in chronic obstructive pulmonary disease on 
cardiovascular system. J Indon Med Assoc. 2011;61(5):225-9

8. Huiart L, Ernst P, Suissa S. Cardiovascular morbidity and mortality in COPD. Chest. 2005;128(4):2640-6.

9. Mills NL, Miller JJ, Anand A, Robinson SD, Frazer GA, Anderson D, et al. Increased arterial stiffness in patients with chronic obstructive pulmonary disease: a mechanism for increased cardiovascular risk. Thorax. 2008;63(4):306-11.

10. Mannino DM, Thorn D, Swensen A, Holguin F. Prevalence and outcomes of diabetes, hypertension and cardiovascular disease in COPD. Eur Respir J. 2008;32(4):962-9.

11. John M, Hoernig S, Doehner W, Okonko DD, Witt C, Anker SD. Anemia and inflammation in COPD. Chest. 2005;127(3):825-9.

12. Yohannes AM, Ershler WB. Anemia in COPD: a systematic review of the prevalence, quality of life, and mortality. Respir Care. 2011;56(5):644-52.

13. Holguin F, Folch E, Redd SC, Mannino DM. Comorbidity and mortality in COPD-related hospitalizations in the United States, 1979 to 2001. Chest. 2005;128(4):2005-11.

14. Graat-Verboom L, van den Borne BE, Smeenk FW, Spruit MA, Wouters EF. Osteoporosis in COPD outpatients based on bone mineral density and vertebral fractures. J Bone Miner Res. 2011;26(3):561-8.

15. Barnes P, Celli B. Systemic manifestations and comorbidities of COPD. Eur Respir J. 2009;33(5):1165-85.

16. de Torres JP, Marín JM, Casanova C, Cote C, Carrizo S, Cordoba-Lanus E, et al. Lung cancer in patients with chronic obstructive pulmonary disease. Am J Respir Crit Care Med. 2011;184(8):913-9.

17. Minai OA, Chaouat A, Adnot S. Pulmonary hypertension in COPD: epidemiology, significance, and management pulmonary vascular disease: the global perspective. Chest. 2010;137(suppl6):S39-S51.

18. Joppa P, Petrasova D, Stancak B, Tkacova R. Systemic inflammation in patients with COPD and pulmonary hypertension. Chest. 2006;130(2):326-33. 